\title{
Bei Typ-1-Diabetikern ist das Risiko deutlich erhöht
}

\author{
Die Meldung über das Auftreten von Ketoazidosen un- \\ ter Gliflozinen hat zu einer gewissen Verunsicherung \\ geführt. Betroffen waren nicht nur Typ-1-Diabetiker, \\ bei denen diese Substanzen gelegentlich off-label ein- \\ gesetzt werden, sondern auch Typ-2-Diabetiker. Doch \\ wie sieht die Datenlage aus? Ist das Risiko bei Typ- \\ 1-Diabetikern höher als bei Typ-2-Diabetikern und wie \\ lässt sich eine solche Komplikation verhindern?
}

Die Einführung der SGLT2-Inhibitoren (Canagliflozin, Dapagliflozin, Empagliflozin) hat das Spektrum der oralen antidiabetischen Therapieoptionen erweitert. In den Zulassungsstudien erwiesen sich diese Substanzen als sehr sicher. Angesichts des innovativen Wirkmechanismus besteht kein erhöhtes Hypoglykämierisiko, da diese Substanzen unabhängig von Insulin wirken. Abgesehen von Genitalinfektionen fand sich im Vergleich zu Placebo kein erhöhtes Risiko für etwaige Nebenwirkungen. Doch im Juni dieses Jahres wurden dem Adverse Event Reporting System der FDA 20 Fälle von Ketoazidosen gemeldet, und zwar nicht nur bei Typ-1-, sondern auch bei Typ-2-Diabetikern, bei denen eine solche Komplikation eigentlich sehr selten ist. Im Unterschied zu typischen Ketoazidosen waren die Blutzuckerspiegel nur leicht erhöht. Das hat auch die EMA veranlasst, das Sicherheitsprofil dieser Substanzklasse nochmals genau zu überprüfen. Auch die Hersteller haben in Informationsbriefen die Ärzte darüber informiert und empfohlen, schon beim geringsten Verdacht auf eine Ketoazidose unter einem Gliflozin die Ketonkörper zu bestimmen.

\section{Sehr selten bei Typ-2-Diabetes}

Die jüngst veröffentlichte EMPA-REG OUTCOME-Studie, in der erstmals für ein Antidiabetikum, nämlich für Empagliflozin, eine die Prognose verbessernde Wirkung nachgewiesen wurde, ergab für diese Substanz eine Häufigkeit bzgl. einer Ketoazidose von $0,1 \%$ [1]. In einer neueren Metaanalyse, bei der die Daten von 17.596 mit Canagliflozin behandelten Typ-2-Diabetikern ausgewertet wurden, lag das Risiko für eine schwere Ketoazidose $<1 \%$ [2]. „Dabei waren meist Patienten betroffen, die Insulin spritzten", so Professor Anne Peters, Los Angeles. Darüberhinaus bestanden prädisponierende Einflüsse, die das Auftreten einer Ketoazidose begünstigten, nämlich eine Infektion oder eine fehlende Compliance bzgl. der Insulintherapie. Als möglicher auslösender Mechanismus wird auch die unter dem SGLT2-Inhibitor gesteigerte Glukagonbildung diskutiert [3].

\section{Bei Typ-1-Diabetikern häufiger}

Für Typ-1-Diabetiker ist die Insulinsubstitution unverzichtbar. Doch 25\% der erwachsenen Typ-1-Diabetiker erreichen damit nicht den $\mathrm{HbA}_{\mathrm{lc}}$-Zielwert $<7 \%$. Dazu kommen Probleme mit dem Körpergewicht, das Hypoglykämierisiko und oft starke Fluktuationen der Blutzuckerwerte. Auch besteht bei Typ-1-Diabtikern immer ein erhöhtes Risiko für eine Ketoazidose, da die betroffenen Patienten kaum noch eigenes Insulin bilden. „Somit besteht durchaus auch beim Typ-1-Diabetes Bedarf für neue

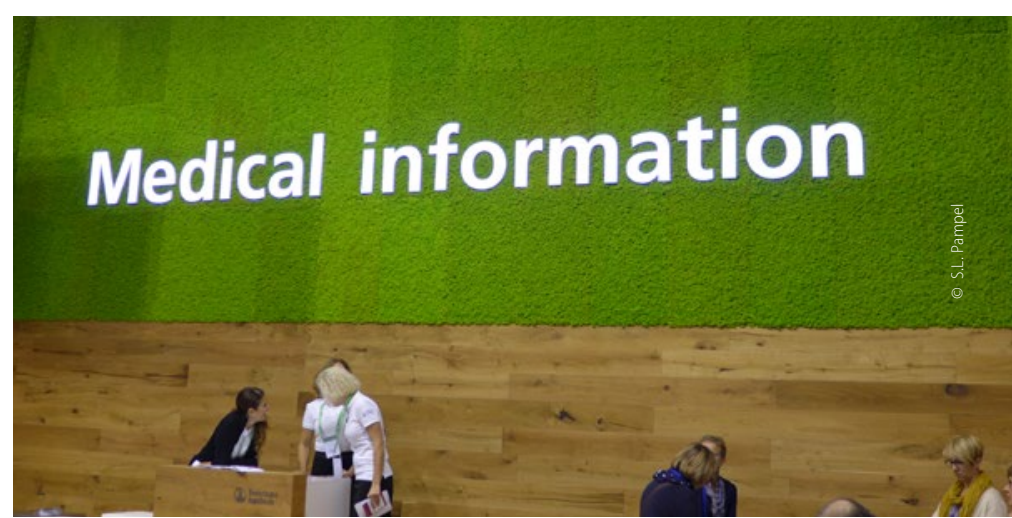

Im Juni 2015 wurden dem Adverse Event Reporting System der FDA 20 Fälle von Ketoazidosen bei Gliflozintherapie gemeldet.

Therapiestrategien, die das Hypoglykämierisiko nicht erhöhen“, so Professor Robert R. Henry, San Diego. Dies sei die Rationale für den Einsatz von Gliflazonen bei Typ-1-Diabetikern, was allerdings ein Off Label Use darstelle.

Im Rahmen einer Phase-2-Studie wurde die Wirksamkeit und Sicherheit von Canagliflozin (in Deutschland nicht mehr verfügbar) in einer Dosierung von $100 \mathrm{mg}$ oder $300 \mathrm{mg}$ bei 351 Typ1-Diabetikern in einem Alter zwischen 25-65 Jahren und einem $\mathrm{HbA}_{1 \mathrm{c}}$-Wert von 7\%-8\% im Vergleich mit Placebo über 18 Wochen untersucht. Der SGLT2-Inhibitor führte zu einer Senkung des $\mathrm{HbA}_{1 \mathrm{c}}$-Wertes und des Körpergewichts ohne Anstieg des Hypoglykämierisikos. Doch die Zahl der Ketoazidosen nahm deutlich zu. Das Risiko für diese Komplikation lag unter $100 \mathrm{mg}$ Canagliflozin bei $5,1 \%$, davon handelte es sich bei $4,3 \%$ um eine schwere Ketoazidose. Unter $300 \mathrm{mg}$ Canagliflozin betrug das Gesamtrisiko 9,4\%, davon waren 5,0\% schwere Ketoazidosen [4]. Bei allen Patienten mit einer schweren Ketoazidose lagen prädisponierende Faktoren vor: Pneumonie, Influenza, Störungen der Insulinpumpe oder unzureichende Compliance bzgl. der Insulininjektion. Auch unter anderen Gliflozinen war das Ketoazidoserisiko bei Typ-1-Diabetikern deutlich erhöht, nämlich $12 \%$ bei Sotagliflozin und 5\% bei Empagliflozin. „Die Gabe eines Gliflozins bei Typ-1-Diabetikern erfordert deshalb eine regelmäßige Bestimmung der Ketonkörper“, so Henry. Der SGLT2-Inhibitor sollte auch nur in einer Dosierung von 25-50\% der niedrigsten Dosis gegeben und die Menge des Basalinsulins nur um 10\% reduziert werden. Bei einem Anstieg der Ketonkörper müsse der SGLT2-Inhibitor sofort abgesetzt werden. Dr. med. Peter Stiefelhagen

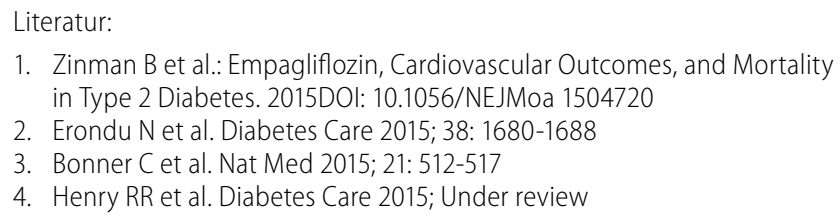

Quelle: Symposium „Update on ketoacidosis with SGLT2 inhibitors“; 18.9.2015; EASD-Jahrestagung, Stockholm 